\title{
Keratin Intermediate Filament Three-Dimensional Arrangements Modeled from TEM Tomograms of Romney Wool Cortical Cells
}

\author{
J. P. Caldwell*, D. N. Mastronarde ${ }^{* *}$, J. L. Woods", W. G. Bryson ${ }^{*}$ \\ *Canesis Network Limited, Private Bag 4749, Christchurch, New Zealand \\ *** University of Colorado, Campus Box 347, Boulder, Colorado, USA
}

Since pre-historic times, humans have used wool and other mammalian fibers for interior textiles (carpets and mats) and apparel (knitted and woven garments). Fiber mechanical studies show keratin intermediate filaments (IFs) are key determinants of textile properties such as intrinsic fiber strength. IFs are the dominant structural component, comprising approximately $60 \%$ of the wool fiber by mass. IFs, $8 \mathrm{~nm}$ in diameter, are arranged longitudinally to the fiber axis in bundles (macrofibrils) located in cortical cells. This paper reveals that the short-range, three-dimensional (3D), ultrastructural arrangements of IFs can be classified into three types each specific to either ortho-, para- or mesocortical cell types. The lateral arrangement of these cortical cell types can be related to variations in fiber curvature. Since the discrete IF arrangements are the main ultrastructural feature distinguishing the cortical cell types (Figure 1) then it is reasonable to assume these arrangements influence fiber curvature. Curvature adds bulk to wool yarns significantly improving the economics of wool production and the performance of wool products.

Electron tomography was applied to reveal IF 3D arrangements and to quantify IF parameters to differentiate cortical cell ultrastructure. Romney wool fibers were stained with osmium tetroxide, embedded in Spurr's resin, then sectioned (purple/gold, $\sim 150 \mathrm{~nm}$ ) before staining with uranyl acetate and lead citrate. Gold fiducials $(15 \mathrm{~nm})$ were applied to carbon coated grids to align tilt images. Ortho-, para-, and mesocortical cell regions were imaged at the Boulder Laboratory for 3D Electron Microscopy of Cells, University of Colorado, on a JEM-1000 HVEM (750 kV, 12,000x magnification). Each sample was tilted from $-60^{\circ}$ to $+60^{\circ}$ at $1.5^{\circ}$ increments relative to the axis of the TEM beam to collect eighty one images $(1.5$ by $1.5 \mu \mathrm{m})$ via CCD camera (Gatan, 1024 x 1024 pixels) of a macrofibrillar region within a cell type. A tomogram was reconstructed from stacked and aligned tilt-series images using IMOD software [1]. Figures 1a, 1b and 1c, represent end-on views of tomographic reconstructions of ortho-, para-, and mesocortical macrofibril regions.

Single macrofibrils were selected for IMOD modeling of IFs. Tilting the tomographic image in the $\mathrm{x}, \mathrm{y}$ or $\mathrm{z}$ plane enabled end-on viewing of IFs. Slicing the tomogram at appropriate depths allowed the tracing of each IF. IFs were modeled in a cartwheel pattern from the macrofibril centre to the periphery. The models (Figure 1) show a series of views from: end-on down-the-filament $\left(0^{\circ}\right.$ macrofibril tilt), intermediate $\left(45^{\circ}\right.$ macrofibril tilt), and side-on $\left(90^{\circ}\right.$ macrofibril tilt). In the orthocortical model, IFs were observed to be right-hand helically tilted in the plane tangential to an imaginary cylinder formed by the IF helix and aligned parallel to the macrofibril axis. For different IFs the helical tilt angle progressively increased from the centre to the peripheral macrofibril boundary to give maximum IF helical angles of $\sim 25^{\circ}$ at distances of 150 to $200 \mathrm{~nm}$ from the macrofibril center. Modeled para- and mesocortical macrofibril regions revealed that the majority of IFs were spatially arranged parallel to the macrofibril axis with small deviations from this arrangement towards the periphery of the modeled regions. Whereas the paracortical IFs appeared pseudo-hexagonally packed with larger IF to IF inter-spacing distances, the mesocortex contained 
hexagonally close-packed IFs. Of importance to fiber flexural behaviour, these IF packing arrangements imply that paracortical IFs may be able to yield to compression forces by buckling. Actual IF helical angles and distances from the central macrofibril axis were obtained using Fiberpitch (a member of the IMOD software suite) and results are presented (Figure 2). The experimentally measured angles of the individual IFs within the three cortical cell types can be incorporated into a computational mathematical model for predicting single fiber mechanical properties such as stiffness [2] and curvature.

\section{References}

[1] Kremer, J. R., et al., 1996; http://bio3d.colorado.edu.

[2] Liu, H. and Bryson W.G. (2002) A three component model of the wool fibre - effects of morphology, elasticity and intermediate filament arrangement on fibre stiffness. J. Text. Inst. 93, Part 1, 121-131.

[3] The authors are grateful to the Foundation for Research, Science and Technology in New Zealand for funding support under Contract WROX004 and for Grant RR00592 to J.R. McIntosh from the National Center for Research Resources, USA, and thank Mary Morphew and Eileen O'Toole for assistance in sample preparation and image collection.

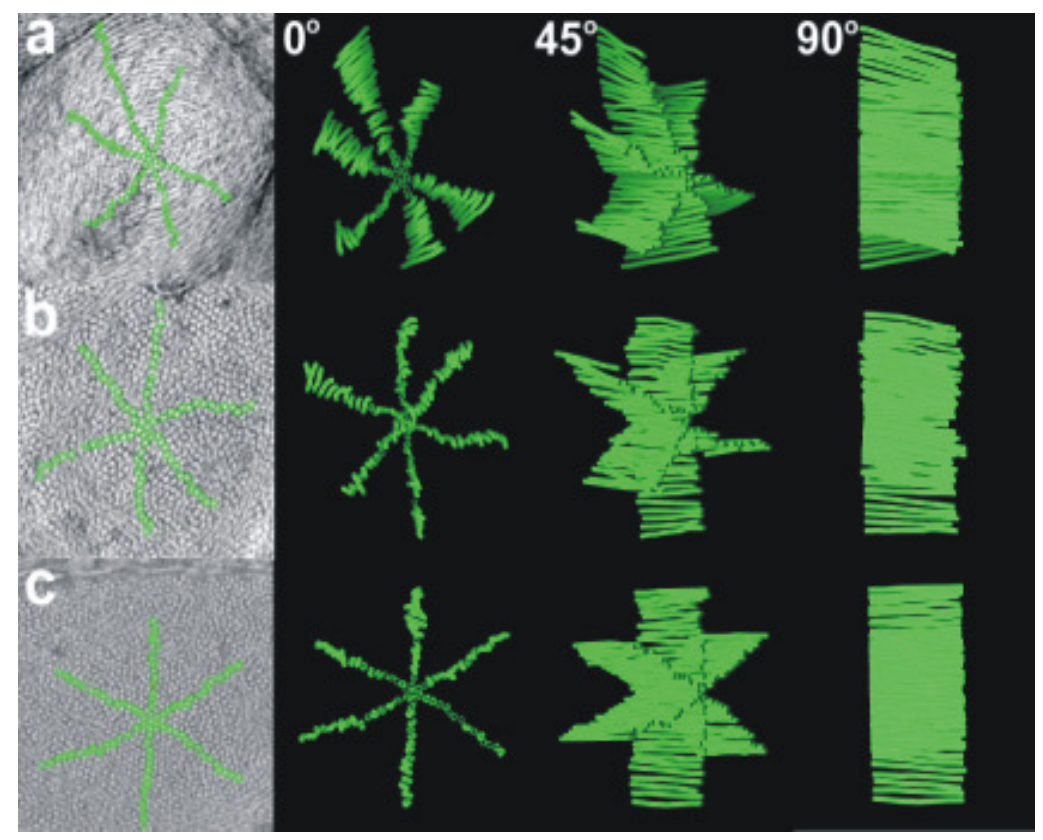

Figure 1. Tomographic views of (a) ortho-, (b) para- and (c) mesocortex macrofibrils (LHS) with corresponding 3D models of IF arrangements tilted at $0^{\circ}, 45^{\circ}$ and $90^{\circ}$ relative to the macrofibril axis.

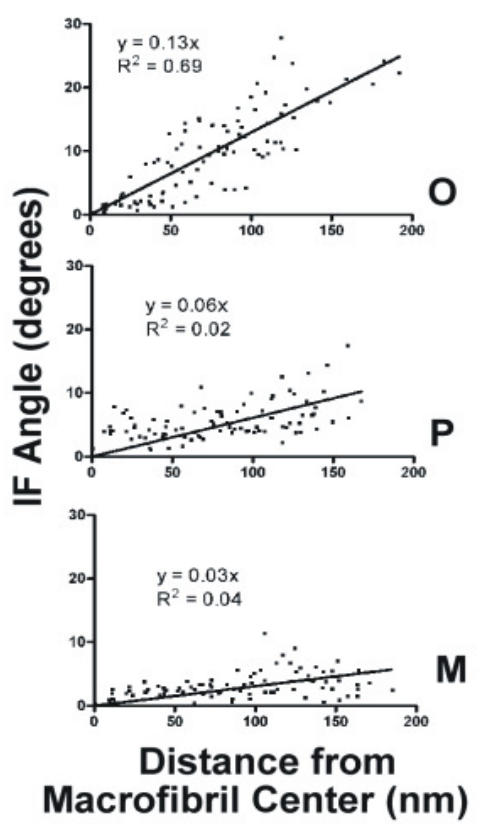

Figure 2. Plots of IF helix angle versus distance from the macrofibril center for the respective ortho-, para- and mesocortical models. 